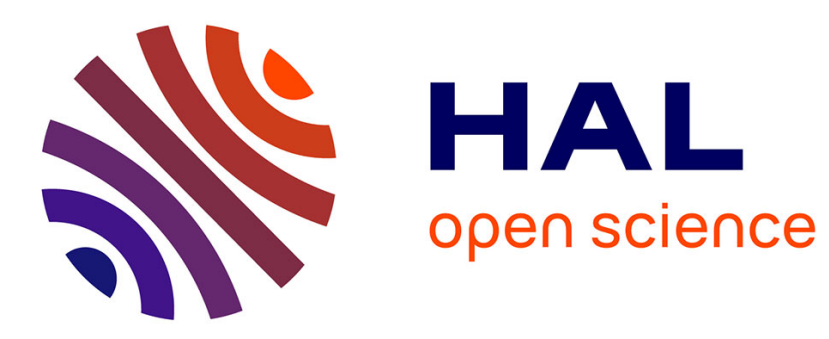

\title{
Recent Achievements on a DGTD Method for Time-Domain Electromagnetics
}

\author{
Hassan Fahs, Loula Fatima Fezoui, Stephane Lanteri, Victorita Dolean, \\ Francesca Rapetti
}

\section{> To cite this version:}

Hassan Fahs, Loula Fatima Fezoui, Stephane Lanteri, Victorita Dolean, Francesca Rapetti. Recent Achievements on a DGTD Method for Time-Domain Electromagnetics. IEEE Transactions on Magnetics, 2010, 46 (8), pp.3061 - 3064. 10.1109/TMAG.2010.2043342 . hal-00600466

\section{HAL Id: hal-00600466 https://hal.science/hal-00600466}

Submitted on 9 Jul 2013

HAL is a multi-disciplinary open access archive for the deposit and dissemination of scientific research documents, whether they are published or not. The documents may come from teaching and research institutions in France or abroad, or from public or private research centers.
L'archive ouverte pluridisciplinaire HAL, est destinée au dépôt et à la diffusion de documents scientifiques de niveau recherche, publiés ou non, émanant des établissements d'enseignement et de recherche français ou étrangers, des laboratoires publics ou privés. 


\title{
Recent achievements on a DGTD method for time-domain electromagnetics
}

\author{
Hassan Fahs, Loula Fezoui, Stéphane Lanteri, Victorita Dolean and Francesca Rapetti
}

\begin{abstract}
We report on results concerning a discontinuous Galerkin time domain (DGTD) method for the solution of Maxwell equations. This DGTD method is formulated on unstructured simplicial meshes (triangles in 2D and tetrahedra in 3D). Within each mesh element, the electromagnetic field components are approximated by an arbitrarily high order nodal polynomial while, in the original formulation of the method, time integration is achieved by a second order Leap-Frog scheme. Here, we discuss about several recent developments aiming at improving the accuracy and the computational efficiency of this DGTD method in view of the simulation of problems involving general domains and heterogeneous media.
\end{abstract}

\section{INTRODUCTION}

Nowadays, a variety of methods exist for the solution of the time domain Maxwell equations ranging from the well established FDTD methods to FETD methods and DGTD methods. In the recent years, there has been an increasing interest in DGTD methods which have been developed on quadrangular/hexahedral [1] and triangular/tetrahedral [2] meshes. In this paper, we report on some recent achievements for improving the accuracy and the computational efficiency of a DGTD method that was originally introduced in [3]. The topics addressed here are concerned with (a) dealing with a non-conforming local refinement of the mesh and a local definition of the approximation order, (b) designing a hybrid explicit-implicit time stepping strategy and, (c) computing on curvilinear domains.

\section{HIGH ORDER DGTD METHOD ON SIMPLICIAL MESHES}

\section{A. Continuous problem}

We consider the Maxwell equations for heterogeneous linear isotropic media. The electric field $\vec{E}(\vec{x}, t)={ }^{t}\left(E_{x}, E_{y}, E_{z}\right)$ and the magnetic field $\vec{H}(\vec{x}, t)={ }^{t}\left(H_{x}, H_{y}, H_{z}\right)$ verify:

$$
\epsilon \partial_{t} \vec{E}-\operatorname{curl} \vec{H}=-\vec{J}, \quad \mu \partial_{t} \vec{H}+\operatorname{curl} \vec{E}=0,
$$

where the symbol $\partial_{t}$ denotes a time derivative and $\vec{J}(\vec{x}, t)$ is a current source term. These equations are set on a bounded polyhedral domain $\Omega$ of $\mathbb{R}^{3}$. The permittivity $\epsilon(\vec{x})$ and the magnetic permeability tensor $\mu(\vec{x})$ are varying in space, timeinvariant and both positive functions. Our goal is to solve system (1) in $\Omega$ with boundary $\partial \Omega=\Gamma_{a} \cup \Gamma_{m}$, where we

H. Fahs, L. Fezoui and S. Lanteri are with INRIA, 06902 Sophia Antipolis, France (e-mail: Hassan.Fahs@gmail.com, Loula.Fezoui@inria.fr, Stephane.Lanteri@inria.fr).

V. Dolean and F. Rapetti are with the Nice/Sophia Antipolis University, J.A. Dieudonné Mathematics Lab., UMR CNRS 6621, 06108 Nice, France (e-mail: \{dolean, frapetti\}@unice.fr).

Manuscript received December 18, 2009. impose the following boundary conditions: $\vec{n} \times \vec{E}=0$ on $\Gamma_{m}$ and $\mathcal{L}(\vec{E}, \vec{H})=\mathcal{L}\left(\vec{E}_{\text {inc }}, \vec{H}_{\text {inc }}\right)$ on $\Gamma_{a}$, where $\Gamma_{m}$ (resp. $\left.\Gamma_{a}\right)$ is the metallic (absorbing) boundary, $\mathcal{L}(\vec{E}, \vec{H})=\vec{n} \times \vec{E}-$ $\sqrt{\frac{\mu}{\varepsilon}} \vec{n} \times(\vec{H} \times \vec{n})$. Here $\vec{n}$ denotes the unit outward normal to $\partial \Omega$ and $\left(\vec{E}_{\text {inc }}, \vec{H}_{\text {inc }}\right)$ is a given incident field. Finally, system (1) is supplemented with initial conditions: $\vec{E}_{0}(\vec{x})=\vec{E}(\vec{x}, t)$ and $\vec{H}_{0}(\vec{x})=\vec{H}(\vec{x}, t)$.

\section{B. Discretization in space}

We consider a partition $\mathcal{T}_{h}$ of $\Omega$ into a set of elements $\tau_{i}$ (triangles in 2D and tetrahedra in 3D) of size $h_{i}$ with boundary $\partial \tau_{i}$ such that $h=\max \left\{h_{i}, \tau_{i} \in \mathcal{T}_{h}\right\}$. For each $\tau_{i}, \epsilon_{i}$ and $\mu_{i}$ are respectively the piecewise constant electric permittivity and magnetic permeability of the medium. For two elements $\tau_{i}$ and $\tau_{k}$ in $\mathcal{T}_{h}$, the intersection $a_{i k}=\tau_{i} \cap \tau_{k}$ is called an interface. For each internal interface $a_{i k}$, we denote by $\vec{n}_{i k}$ the unitary normal vector, oriented from $\tau_{i}$ to $\tau_{k}$. For boundary interfaces, the index $k$ corresponds to a fictitious element outside $\Omega$. Let $\mathcal{F}_{h}^{i}$ be the set of interior interfaces of $\mathcal{T}_{h}, \mathcal{F}_{h}^{m}$ and $\mathcal{F}_{h}^{a}$ the sets of metallic and absorbing boundary interfaces of $\mathcal{T}_{h}$, and let $\mathcal{F}_{h}=\mathcal{F}_{h}^{i} \cup \mathcal{F}_{h}^{m} \cup \mathcal{F}_{h}^{a}$. We denote by $\mathcal{V}_{i}$ the set of indices of the elements which are neighbors of $\tau_{i}$ (having an interface in common). In the following, to simplify the presentation, we set $\vec{J}=0$. We seek approximate solutions to (1) in $V_{p_{i}}\left(\mathcal{T}_{h}\right)=$ $\left\{\vec{v} \in L^{2}(\Omega)^{3}: \vec{v}_{\mid \tau_{i}} \in\left(\mathbb{P}_{p_{i}}\left(\tau_{i}\right)\right)^{3} \quad \forall \tau_{i} \in \mathcal{T}_{h}\right\}$ where $\mathbb{P}_{p_{i}}\left(\tau_{i}\right)$ denotes the space of nodal polynomial functions of degree at most $p_{i}$ inside the element $\tau_{i}$.

Following the discontinuous Galerkin approach, the electric and magnetic fields inside each finite element are seeked for as linear combinations of linearly independent basis vector fields $\vec{\varphi}_{i j}, 1 \leq j \leq d_{i}$, where $d_{i}$ denotes the local number of degrees of freedom inside $\tau_{i}: \overrightarrow{\mathbf{E}}_{i} \equiv \overrightarrow{\mathbf{E}}_{h \mid \tau_{i}}=\sum_{j} E_{i j} \vec{\varphi}_{i j}$ and $\overrightarrow{\mathbf{H}}_{i} \equiv \overrightarrow{\mathbf{H}}_{h \mid \tau_{i}}=\sum_{j} H_{i j} \vec{\varphi}_{i j}$. The approximate fields are allowed to be discontinuous across element boundaries. For such a discontinuous field $\overrightarrow{\mathbf{U}}_{h}$, we define its average $\left\{\overrightarrow{\mathbf{U}}_{h}\right\}_{i k}$ through any internal interface $a_{i k}$, as $\left\{\overrightarrow{\mathbf{U}}_{h}\right\}_{i k}=\left(\overrightarrow{\mathbf{U}}_{i \mid a_{i k}}+\right.$ $\left.\overrightarrow{\mathbf{U}}_{k \mid a_{i k}}\right) / 2$. Dot-multiplying (1) by $\vec{\varphi} \in \mathcal{P}_{i}=\operatorname{Span}\left(\overrightarrow{\varphi_{i j}}, 1 \leq\right.$ $\left.j \leq d_{i}\right)$, integrating over $\tau_{i}$ and integrating by parts, yields:

$$
\left\{\begin{array}{l}
\int_{\tau_{i}} \vec{\varphi} \cdot \epsilon_{i} \partial_{t} \overrightarrow{\mathbf{E}}=\int_{\tau_{i}} \operatorname{curl} \vec{\varphi} \cdot \overrightarrow{\mathbf{H}}-\int_{\partial \tau_{i}} \vec{\varphi} \cdot(\overrightarrow{\mathbf{H}} \times \vec{n}), \\
\int_{\tau_{i}} \vec{\varphi} \cdot \mu_{i} \partial_{t} \overrightarrow{\mathbf{H}}=-\int_{\tau_{i}} \operatorname{curl} \vec{\varphi} \cdot \overrightarrow{\mathbf{E}}+\int_{\partial \tau_{i}} \vec{\varphi} \cdot(\overrightarrow{\mathbf{E}} \times \vec{n}) .
\end{array}\right.
$$

In Eq. (2), we now replace the exact fields $\overrightarrow{\mathbf{E}}$ and $\overrightarrow{\mathbf{H}}$ by the approximate fields $\overrightarrow{\mathbf{E}}_{i}$ and $\overrightarrow{\mathbf{H}}_{i}$ in order to evaluate volume integrals. For integrals over $\partial \tau_{i}$, a specific treatment 
must be introduced since the approximate fields are discontinuous through element faces, leading to the definition of a numerical flux. We choose to use a fully centered numerical flux, i.e. $\forall i, \forall k \in \mathcal{V}_{i}, \overrightarrow{\mathbf{E}}_{\mid a_{i k}} \simeq\left\{\overrightarrow{\mathbf{E}}_{h}\right\}_{i k}, \overrightarrow{\mathbf{H}}_{\mid a_{i k}} \simeq\left\{\overrightarrow{\mathbf{H}}_{h}\right\}_{i k}$. Evaluating the surface integrals in (2) using the centered numerical flux, and re-integrating by parts yields:

$$
\left\{\begin{aligned}
\int_{\tau_{i}} \vec{\varphi} \cdot \epsilon_{i} \partial_{t} \overrightarrow{\mathbf{E}}_{i} & =\frac{1}{2} \int_{\tau_{i}}\left(\operatorname{curl} \vec{\varphi} \cdot \overrightarrow{\mathbf{H}}_{i}+\operatorname{curl} \overrightarrow{\mathbf{H}}_{i} \cdot \vec{\varphi}\right) \\
& -\frac{1}{2} \sum_{k \in \mathcal{V}_{i}} \int_{a_{i k}} \vec{\varphi} \cdot\left(\overrightarrow{\mathbf{H}}_{k} \times \vec{n}_{i k}\right), \\
\int_{\tau_{i}} \vec{\varphi} \cdot \mu_{i} \partial_{t} \overrightarrow{\mathbf{H}}_{i} & =-\frac{1}{2} \int_{\tau_{i}}\left(\operatorname{curl} \vec{\varphi} \cdot \overrightarrow{\mathbf{E}}_{i}+\operatorname{curl} \overrightarrow{\mathbf{E}}_{i} \cdot \vec{\varphi}\right) \\
& +\frac{1}{2} \sum_{k \in \mathcal{V}_{i}} \int_{a_{i k}} \vec{\varphi} \cdot\left(\overrightarrow{\mathbf{E}}_{k} \times \vec{n}_{i k}\right) .
\end{aligned}\right.
$$

The metallic boundary condition on a boundary interface $a_{i k} \in \Gamma_{m}$ ( $k$ in the element index of the fictitious neighboring element) is weakly imposed, in the sense that traces of fictitious fields $\overrightarrow{\mathbf{E}}_{k}$ and $\overrightarrow{\mathbf{H}}_{k}$ are used for the computation of numerical fluxes for the boundary element $\tau_{i}$. More precisely, we set $\overrightarrow{\mathbf{E}}_{k \mid a_{i k}}=-\overrightarrow{\mathbf{E}}_{i \mid a_{i k}}$ and $\overrightarrow{\mathbf{H}}_{k \mid a_{i k}}=\overrightarrow{\mathbf{H}}_{i \mid a_{i k}}$. A similar approach is applied to the numerical treatment of the absorbing boundary condition which is taken into account through the use of a fully upwind numerical flux for the evaluation of the corresponding boundary integral over $a_{i k} \in \Gamma_{a}$ (see [3] for more details). Let us denote by $\mathbf{E}_{i}$ and $\mathbf{H}_{i}$ respectively the column vectors $\left(E_{i l}\right)_{1 \leq l \leq d_{i}}$ and $\left(H_{i l}\right)_{1 \leq l \leq d_{i}}$. Eq. (3) can be rewritten as:

$$
\left\{\begin{array}{l}
M_{i}^{\epsilon} \frac{d \mathbf{E}_{i}}{d t}=K_{i} \mathbf{H}_{i}-\sum_{k \in \mathcal{V}_{i}} S_{i k} \mathbf{H}_{k}, \\
M_{i}^{\mu} \frac{d \mathbf{H}_{i}}{d t}=-K_{i} \mathbf{E}_{i}+\sum_{k \in \mathcal{V}_{i}} S_{i k} \mathbf{E}_{k},
\end{array}\right.
$$

where the symmetric positive definite mass matrices $M_{i}^{\sigma}(\sigma$ stands for $\epsilon$ or $\mu$ ), the symmetric stiffness matrix $K_{i}$ and the symmetric interface matrix $S_{i k}$ (all of size $d_{i} \times d_{i}$ ) write:

$$
\begin{aligned}
\left(M_{i}^{\sigma}\right)_{j l} & =\sigma_{i} \int_{\tau_{i}}{ }^{t} \vec{\varphi}_{i j} \cdot \vec{\varphi}_{i l} \\
\left(K_{i}\right)_{j l} & =\frac{1}{2} \int_{\tau_{i}}^{t} \vec{\varphi}_{i j} \cdot \operatorname{curl} \vec{\varphi}_{i l}+{ }^{t} \vec{\varphi}_{i l} \cdot \operatorname{curl} \vec{\varphi}_{i j} \\
\left(S_{i k}\right)_{j l} & =\frac{1}{2} \int_{a_{i k}}^{t} \vec{\varphi}_{i j} \cdot\left(\vec{\varphi}_{k l} \times \vec{n}_{i k}\right)
\end{aligned}
$$

\section{Time discretization}

The set of local system of ordinary differential equations for each $\tau_{i}$ (4) can be formally transformed in a global system. To this end, we suppose that all electric (resp. magnetic) unknowns are gathered in a column vector $\mathbb{E}$ (resp. $\mathbb{H})$ of size $d_{g}=\sum_{i=1}^{N_{t}} d_{i}$ where $N_{t}$ stands for the number of elements in $\mathcal{T}_{h}$. Then system (4) can be rewritten as:

$$
\left\{\begin{array}{l}
\mathbb{M}^{\epsilon} \frac{d \mathbb{E}}{d t}=\mathbb{K} \mathbb{H}-\mathbb{A} \mathbb{H}-\mathbb{B} \mathbb{H}+\mathbb{C}_{E} \mathbb{E}, \\
\mathbb{M}^{\mu} \frac{d \mathbb{H}}{d t}=-\mathbb{K} \mathbb{E}+\mathbb{A} \mathbb{E}-\mathbb{B E}+\mathbb{C}_{H} \mathbb{H},
\end{array}\right.
$$

where we have the following definitions and properties:

- $\mathbb{M}^{\epsilon}, \mathbb{M}^{\mu}$ and $\mathbb{K}$ are $d_{g} \times d_{g}$ block diagonal matrices with diagonal blocks equal to $M_{i}^{\epsilon}, M_{i}^{\mu}$ and $K_{i}$ respectively. $\mathbb{M}^{\epsilon}$ and $\mathbb{M}^{\mu}$ are symmetric positive definite matrices, and $\mathbb{K}$ is a symmetric matrix.

- $\mathbb{A}$ is also a $d_{g} \times d_{g}$ block sparse matrix, whose non-zero blocks are equal to $S_{i k}$ when $a_{i k} \in \mathcal{F}_{h}^{i}$. Since $\vec{n}_{k i}=$ $-\vec{n}_{i k}$, it can be checked that $\left(S_{i k}\right)_{j l}=\left(S_{k i}\right)_{l j}$ and then $S_{k i}={ }^{t} S_{i k}$; thus $\mathbb{A}$ is a symmetric matrix.

- $\mathbb{B}$ is a $d_{g} \times d_{g}$ block diagonal matrix, whose non-zero blocks are equal to $S_{i k}$ when $a_{i k} \in \mathcal{F}_{h}^{m}$. In that case, $\left(S_{i k}\right)_{j l}=-\left(S_{i k}\right)_{l j}$; thus $\mathbb{B}$ is a skew-symmetric matrix.

- $\mathbb{C}_{E}$ and $\mathbb{C}_{H}$ are $d_{g} \times d_{g}$ block diagonal matrices associated to boundary integral terms for $a_{i k} \in \mathcal{F}_{h}^{a}$.

Let $\mathbb{S}=\mathbb{K}-\mathbb{A}-\mathbb{B}$; the system (6) rewrites as:

$$
\mathbb{M}^{\epsilon} \frac{d \mathbb{E}}{d t}=\mathbb{S H}+\mathbb{C}_{E} \mathbb{E}, \quad \mathbb{M}^{\mu} \frac{d \mathbb{H}}{d t}=-{ }^{t} \mathbb{S E}+\mathbb{C}_{H} \mathbb{H} .
$$

In [3], the semidiscrete system (6) is time integrated using a second-order Leap-Frog scheme and it is proved that the resulting DGTD- $\mathbb{P}_{p_{i}}$ method is stable under the CFL-like condition.

\section{NON-CONFORMING DGTD METHOD}

One of the distinguishing features of a DGTD method is that it can easily accommodate a non-conforming locally refined mesh (i.e. $h$-refinement) as well as a local definition of the approximation order (i.e. $p$-enrichment), or of both of them in the context of a $h p$-adaptive solution strategy. In [4] we have reported on the results of a preliminary investigation of a $h$-refinement non-conforming DGTD method, by mainly concentrating on stability issues. Thereafter, this initial study has progressed towards the development of a $h p$-like DGTD$\mathbb{P}_{p_{i}}$ method combining $h$-refinement and $p$-enrichment, in the context of the solution of the 2D Maxwell equations [5]. Here, we illustrate the capabilities of this method by considering the simulation of the scattering of a plane wave $(\mathrm{F}=300 \mathrm{MHz})$ by a multilayered cylinder. Each layer consists of a dielectric nonmagnetic material, with $\epsilon_{i}>1$ for $2 \leq i \leq 5$ and $\epsilon_{1}=\epsilon_{6}=1$ (relative values).

To assess the effectiveness of the proposed non-conforming DGTD- $\mathbb{P}_{p_{i}}$ method, we first construct a conforming mesh consisting of 14401 nodes and 28560 triangles and we use different conforming DGTD- $\mathbb{P}_{p}$ methods (i.e. with a spatially uniform interpolation order $p$ ). Then, a non-conforming mesh is obtained by locally refining a coarse conforming mesh where the level of refinement depends on the local wavelength in each region. The resulting non-conforming mesh consists of 27640 triangles and 14441 nodes in which 920 are hanging nodes. For this non-conforming mesh, we assign to each layer a polynomial degree $p_{i}$ based on a simple geometrical criterion. Results are shown on Fig. 1 in terms of the $x$-wise 1D distribution along $y=0.0 \mathrm{~m}$ of the $E_{z}$ component where the bottom figure displays the approximate solutions associated to two configurations of the non-conforming DGTD- $\mathbb{P}_{p_{i}}$ method. One can observe that the proposed non-conforming DGTD method treats very well the steep variations of the field at the 
material interfaces. In order to compare further the conforming and non-conforming methods, we estimate the numerical error based on a reference solution computed on a highly refined conforming mesh. For the conforming DGTD- $\mathbb{P}_{2}$ method, the $L^{2}$ error on $E_{z}$ is equal to $1.3 \%$ and the simulation time is $5 \mathrm{~h} 43 \mathrm{mn}$ (simulations have been carried out on a workstation equipped with an Intel Pentium $\mathrm{M} 1.7 \mathrm{GHz}$ and $1 \mathrm{~GB}$ of RAM) while, for the non-conforming DGTD- $\mathbb{P}_{4,3,2,1,0,2}$ method (where the integer indices represent the polynomial degree within each of the six layers) the corresponding figures are $1.7 \%$ and $12 \mathrm{mn}$.
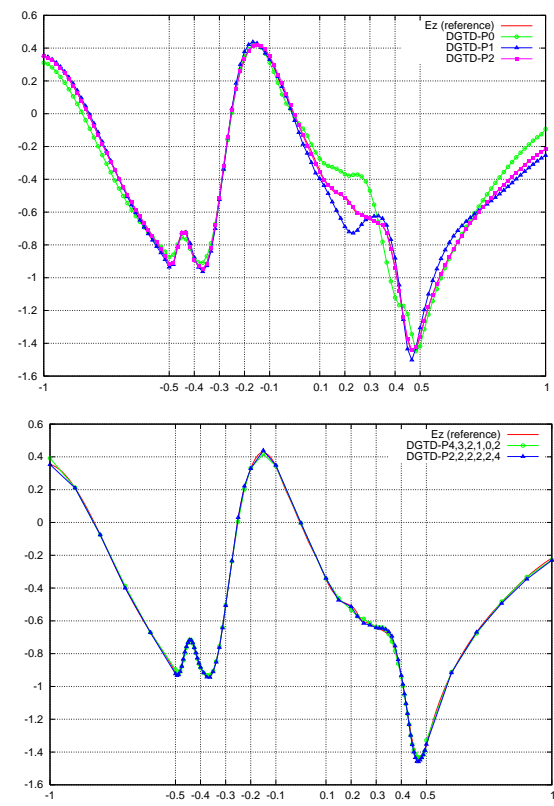

Fig. 1. Scattering of a plane wave by a multilayered cylinder. 1D distribution of $E_{z}$ along $y=0$. Conforming (top) and non-conforming (bottom) DGTD$\mathbb{P}_{p_{i}}$ methods.

\section{HYBRID EXPLICIT-IMPLICIT DGTD METHOD}

Existing DGTD methods generally rely on explicit time integration schemes and are thus constrained by a stability condition that can be very restrictive on locally refined meshes, and when the local approximation relies on high order polynomial interpolation. An implicit time integration scheme is a natural way to obtain a time domain method which is unconditionally stable, but at the expense of the inversion of a global linear system at each time step. A more viable approach consists in applying an implicit time integration scheme locally in the refined regions of the mesh, while preserving an explicit time scheme in the complementary part. Such an hybrid explicit-implicit DGTD method has been proposed by Piperno in [6]. In this method, the elements of the mesh are assumed to be partitioned into two subsets, $\mathcal{S}_{i}$ and $\mathcal{S}_{e}$, on the basis of an appropriate geometrical criterion. Then, the elements of $\mathcal{S}_{i}$ are handled using a Crank-Nicolson scheme while those of $\mathcal{S}_{e}$ are time advanced using a variant of the classical Leap-Frog scheme known as the Verlet method (see [6] for more details). We have recently completed a stability analysis of this method and subsequently implemented it for the solution of the 2D and 3D time domain Maxwell equations discretized in space by a high order conforming DGTD- $\mathbb{P}_{p}$ method on unstructured simplicial meshes [7]. The effectiveness of the resulting hybrid explicit-implicit DGTD$\mathbb{P}_{p}$ method is demonstrated here by considering the simulation of the propagation of an electromagnetic wave emitted by a localized source in a heterogeneous geometrical model of head tissues (see Fig. 2). The underlying tetrahedral mesh consists of 61358 vertices and 366208 elements. The non-uniformity of this mesh can be assessed by evaluating the ratio between the maximum and minimum values of the local time step which is approximately equal to 135 in the present case. For the particular choice of geometric criterion adopted for this simulation, the distribution of mesh tetrahedra is such that $\left|\mathcal{S}_{e}\right|=5142$ and $\left|\mathcal{S}_{e}\right|=361066$ and the implicit elements are time advanced with a global time step which is approximately 4.7 times larger than the smallest time step of the mesh. The linear system of equations associated to the implicit elements is solved using an optimized sparse direct solver and the factorization of the implicit matrix is performed once for all before entering the time stepping loop. The simulations have been carried out on a workstation equipped with an Intel Xeon $2.3 \mathrm{GHz}$ and $16 \mathrm{~GB}$ of RAM. The simulation time for the fully explicit DGTD- $\mathbb{P}_{1}$ method is $14 \mathrm{~h} 22 \mathrm{mn}$ for a total of 42940 time steps, while the corresponding time for the hybrid explicit-implicit DGTD- $\mathbb{P}_{1}$ method is $1 \mathrm{~h} 49 \mathrm{mn}$ for a total of 2780 time steps. The memory overhead induced by the use a sparse direct solver is $774 \mathrm{MB}$ and the time for the factorization of the implicit matrix is $98 \mathrm{sec}$.

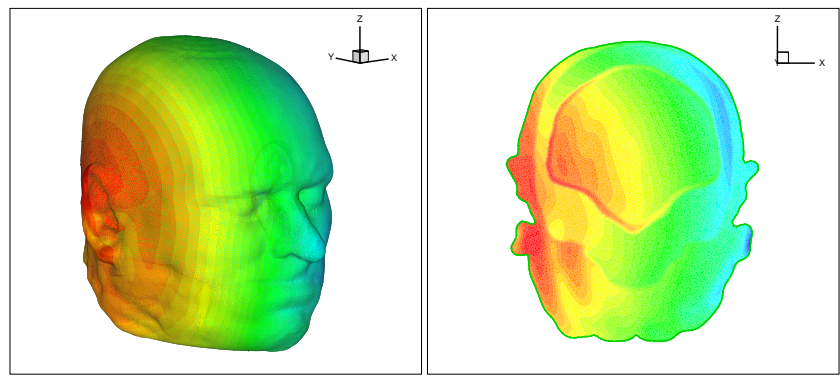

Fig. 2. Head tissues exposure to an electromagnetic wave emitted from a localized source. Contour lines of the normalized SAR in log scale.

\section{DGTD METHOD ON CURVILINEAR DOMAINS}

When designing a high order discretization method, the relevance of an accurate representation of the domain and its boundary has been pointed out by several authors (see for example [8] in the context of a DG method for compressible flow problems). In the basic implementation of the DGTD method described in section II-B, an affine transformation is assumed for the mapping of the reference element to the physical element when computing the elementary integrals (5). Since the Jacobian of the affine mapping is constant, these integrals (i.e. matrices) can be precomputed and stored for the reference element, once for all prior to the time stepping loop. In order to maintain high order accuracy when dealing with curvilinear geometries, an adapted technique has been considered which consists in three ingredients: (a) an isoparametric map for curved elements, (b) a proper numerical 
integration scheme for the evaluation of the matrices (5) which are now stored for each candidate element and, (c) a geometric transformation for the boundaries (edges in 2D and faces in 3D) of the curved elements. To illustrate the benefits of using this isoparametric technique, we consider a circular PEC resonator problem. In order to check the accuracy and the convergence properties of the proposed methodology we present computations with polynomial up de degree $p=4$ in the context of a conforming DGTD- $\mathbb{P}_{p}$ method coupled to a fourth-order Leap-Frog scheme [9], and with affine, quadratic, and cubic mapping from the reference element to the real curved elements. The various computations have been performed on four successively refined non-uniform grids whose characteristics are summarized in Tab. I. Fig. 3 shows the convergence graphs as a function of the square root of the number of degrees of freedom (\#DOF), while the corresponding global $h$-convergence rates are given in Tab. II. The convergence rates obtained with the affine map are bounded by 2 , while those obtained using the quadratic and cubic maps are bounded by 3 and 3.5 respectively. It is clear that the solution accuracy of high degree $p$ is limited by the geometrical error, and that the geometrical error converges at about the same rate as the field error of $p=1$. The errors and the corresponding $p$-convergence rates are given in Table III. The affine map leads to zeroth-order accuracy for $p \geq 2$, while the quadratic and cubic maps achieve exponential convergence.

TABLE I

CHARACTERISTICS OF GRIDS USED FOR THE CIRCULAR PEC RESONATOR.

\begin{tabular}{lcccc}
\hline Mesh number & 1 & 2 & 3 & 4 \\
\hline \hline \# nodes & 51 & 201 & 801 & 3201 \\
\# elements & 80 & 360 & 1520 & 6240 \\
\# curved elements & 10 & 20 & 40 & 80 \\
\hline
\end{tabular}

TABLE II

ASYMPTOTIC CONVERGENCE RATES FOR $h$-REFINEMENT.

\begin{tabular}{|c|c|c|c|}
\hline & Affine map & Quadratic map & Cubic map \\
\hline$p$ & Rate & Rate & Rate \\
\hline 1 & 2.00 & 2.01 & 2.02 \\
\hline 2 & 2.00 & 2.03 & 2.05 \\
\hline 3 & 2.00 & 2.90 & 3.01 \\
\hline 4 & 2.00 & 2.95 & 3.47 \\
\hline
\end{tabular}

TABLE III

$L^{2}$ ERRORS AFTER 10 PERIODS AND CONVERGENCE RATES $r(p)$ FOR $p$-REFINEMENT.

\begin{tabular}{|c|c|c|c|c|c|c|}
\hline \multirow{2}{*}{$p$} & \multicolumn{2}{|c|}{ Affine map } & \multicolumn{2}{|c|}{ Quadratic map } & \multicolumn{2}{|c|}{ Cubic map } \\
\hline & Error & $r(p)$ & Error & $r(p)$ & Error & $r(p)$ \\
\hline$\overline{1}$ & $7.00 \mathrm{E}-02$ & - & $5.18 \mathrm{E}-02$ & - & $5.04 \mathrm{E}-02$ & - \\
\hline 2 & $1.76 \mathrm{E}-02$ & 1.99 & $7.94 \mathrm{E}-04$ & 6.03 & $6.62 \mathrm{E}-04$ & 6.25 \\
\hline 3 & $1.75 \mathrm{E}-02$ & 0.01 & 4.70E-05 & 6.97 & $3.19 \mathrm{E}-05$ & 7.48 \\
\hline 4 & $1.75 \mathrm{E}-02$ & 0.00 & $5.41 \mathrm{E}-06$ & 7.51 & $3.11 \mathrm{E}-06$ & 8.09 \\
\hline
\end{tabular}

\section{CONCLUSION}

We have described several enhancements to a DGTD method in view of the solution of large-scale time domain electromagnetic wave propagation problems involving general domains and heterogeneous media. Future works will aim at the development of $h$-refinement and $p$-enrichment strategies
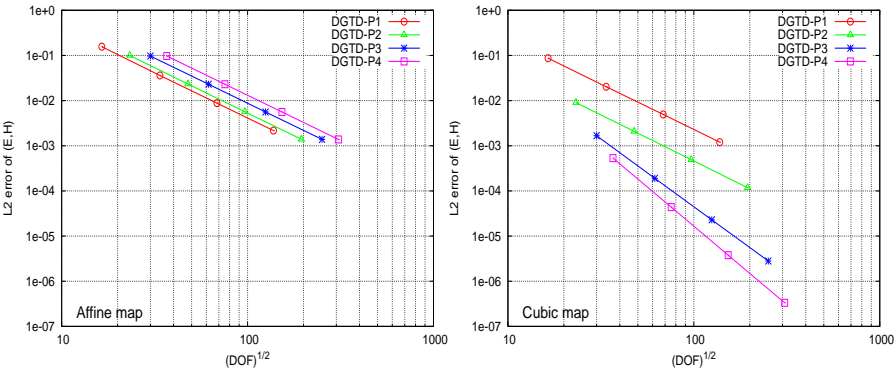

Fig. 3. Circular PEC resonator: $h$-convergence of the DGTD- $\mathbb{P}_{p}$ method. $L^{2}$ error after 2 periods as a function of the square root of \#DOF.

in the 3D case and the extension of the hybrid explicit-implicit time integration scheme to fourth order accuracy.

\section{REFERENCES}

[1] G. Cohen, X. Ferriéres, and S. Pernet, "A spatial high order hexahedral discontinuous Galerkin method to solve Maxwell's equations in timedomain," J. Comput. Phys., vol. 217, no. 2, pp. 340-363, 2006.

[2] J. Hesthaven and T. Warburton, "Nodal high-order methods on unstructured grids. I. Time-domain solution of Maxwell's equations," J. Comput. Phys., vol. 181, no. 1, pp. 186-221, 2002.

[3] L. Fezoui, S. Lanteri, S. Lohrengel, and S. Piperno, "Convergence and stability of a discontinuous Galerkin time-domain method for the 3D heterogeneous Maxwell equations on unstructured meshes," ESAIM: Math. Model. Numer. Anal., vol. 39, no. 6, pp. 1149-1176, 2005.

[4] H. Fahs, L. Fezoui, S. Lanteri, and F. Rapetti, "Preliminary investigation of a non-conforming discontinuous Galerkin method for solving the timedomain Maxwell equations," IEEE Trans. Magn., vol. 44, no. 6, pp. 1254 1257, 2008.

[5] H. Fahs, "Development of a $h p$-like discontinuous Galerkin time-domain method on non-conforming simplicial meshes for electromagnetic wave propagation," Int. J. Numer. Anal. Model., vol. 6, no. 2, pp. 193-216, 2009.

[6] S. Piperno, "Symplectic local time stepping in non-dissipative DGTD methods applied to wave propagation problem," ESAIM: Math. Model. Num. Anal., vol. 40, no. 5, pp. 815-841, 2006.

[7] V. Dolean, H. Fahs, L. Fezoui, and S. Lanteri, "Locally implicit discontinuous Galerkin method for time domain electromagnetics," INRIA, Reserach Report RR-6990, 2009. [Online]. Available: http://hal.inria.fr/inria-00403741

[8] F. Bassi and S. Rebay, "High-order accurate discontinuous finite element solution of the 2D Euler equations," J. Comput. Phys., vol. 138, pp. 252 285, 1997.

[9] H. Fahs and S. Lanteri, "A high-order non-conforming discontinuous Galerkin method for time-domain electromagnetics," J. Comput. Appl. Math., vol. 234, no. 4, pp. 1088-1096, 2010. 